\title{
The Comprehensive ICP (CICP) Algorithm and its Application to the Multidmoal Registration of 3D Surfaces of the Heart
}

\author{
Ahmad Almhdie ${ }^{1,4}$, Christophe Léger ${ }^{1}$, Mohamed Deriche ${ }^{2}$, Long-Dang Nguyen ${ }^{3}$ and Roger Lédée ${ }^{1}$ \\ ${ }^{1}$ LESI, Polytech'Orléans, Université d'Orléans, , France \\ ${ }^{2}$ King Fahd University of Petroleum \& Minerals, Saudi Arabia \\ ${ }^{3}$ Centre hospitalier d'Orléans, Orléans, France \\ ${ }^{4}$ University of Sebha, Libya \\ SEISME, Polytech'Orléans, Université d'Orléans, 12 rue de Blois, 45067 Orléans cedex 02, France \\ Phone: (33) 238494563 Fax: (33) 238417245 \\ E-mails: mderiche@kfupm.edu.sa, \{Ahmad.Almhdie, Christophe.Leger\}@univ-orleans.fr
}

Keywords: Surface comparison, multimodal registration, surface reconstruction, Left ventricle of heart.

\begin{abstract}
Image registration is a valuable technique for medical diagnosis and treatment. In this paper, we present an enhanced implementation of the popular iterative closest point (ICP) algorithm developed for the registration of 3D free-form closed surfaces, based on the use of a look up matrix for finding the best correspondence pairs. The algorithm, called Comprehensive ICP (CICP) algorithm, is then successfully applied for comparing two sequences of 3D surfaces of the left ventricle of the heart, obtained from two different modalities: 4D Echography and Gated SPECT. The results show a good correspondence between the reconstructed sequence from 4D Echography and the reference Gated SPECT sequence. This study can be extended to comparing different medical reconstructions of sphere-like shaped organs collected from different modalities.
\end{abstract}

\section{INTRODUCTION}

The signal and image processing community has recently been paying more attention to integrated approaches for analyzing multi-modal signals/images. In particular, the simultaneous analysis of medical images obtained from different modalities can be very useful in the accurate diagnosis of diseases. But also other applications, such as audio-video processing, can benefit from integrating different signals which can physically be of different nature and to explore their mutual relationship.

In the case of analysis and comparison of medical surfaces, three main aspects should particularly be taken into account. The first aspect relates to the registration of surfaces which are generally not acquired with the same orientation and hence must be realigned in order to be useful to physicians. The second aspect is related to the interpolation of incomplete 3D surfaces, a step necessary to compare and visualize data which might present irregular sampling or different resolutions. The third aspect is related to the robustness of registration algorithms used. By combining various views of an organ, it is possible to compare or integrate the information obtained starting from one or more modalities in order to improve the medical diagnosis.

The work proposed here was initially proposed to validate the reconstructions of an imaging technique developed at the Laboratory of Electronics, Signals, Images (LESI) at the University of Orléans. The new method, called LV4D (for Left Ventricle in 4 Dimensions (3 dimensions + time) provides a representation of the temporal and spatial deformations of the left ventricle of the heart starting from ultrasonic (US) images acquired during a single cardiac cycle [1]. In order to validate the reconstructions produced by the LV4D method, the LV4D surfaces needs to be compared to the reference volumes. In this study, gated SPECT was chosen as the reference modality, since it is used widely in medicine to produce beating LV surfaces.

\section{SURFACE REGISTRATION}

The original iterative closest point algorithm (denoted OICP in this paper), proposed by Besl and McKey [2], is one of the most widespread algorithms used for 3D data registration $[3,4]$. It is particularly valuable in the case of medical imaging registration, since it addresses situations where only few data points are available. This is particularly the case of NM images of the LV of the heart, where images are usually noisy and only 64 by 64 pixels in resolution. In this case, all the data of the targeted organ should be taken into consideration to ensure the maximum use of available information.
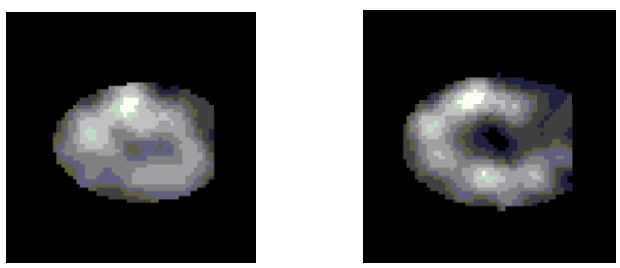

Fig. 1. Example of NM slides of the LV of heart.

The OICP algorithm is designed to register rigid data sets. So, it is well adapted for the medical application addressed in this paper. In fact, we focus more on surface comparison than on surface fitting, which means that only parameter estimation of rigid transformation is required, without introducing deformation or modification of the organs (only translation, rotation and/or scaling are allowed).

The original ICP, algorithm is an iterative algorithm that consists generally of three main steps. The objective is to 
find a transformation that properly matches the scene surface $(\mathbf{M})$ with the reference one $(\mathbf{P})$ and minimizes the distance between these.

The first step in the OICP algorithm is based on the search for pairs of nearest points between the two sets. The second step includes the estimation of the optimal rigid transformation that aligns the two data sets. Then, in the last step, the rigid transformation is applied to the points of the scene data. The procedure is iterated until convergence is achieved.

Widespread interest in 3D surface registration using the OICP algorithm has motivated the scientific community to propose new techniques for enhancing the different steps of the original algorithm. Many variants have been developed for speeding up the convergence and/or improving the performance of the different phases of the algorithm. A good review of these variants can be found in [5]. Additional features, such as curvature and moment invariants, have also been used to improve the correspondence search.

A drawback of the previously proposed ICP variants resides in the difficulty in producing a one to one match between the points of the two data sets to be registered. This leads to multiple assignment problems that we propose to solve by introducing a new matching process based on a complete lookup matrix.

\section{THE MULTIPLE ASSIGNMENT PROBLEM}

In previous variants of the OICP algorithm, the search procedures for corresponding pairs of points were based on a line-by-line (vector) search within a P-M distance matrix described in Table 1. In this table, the $1^{\text {st }}$ row describes the indices of the reference data points and the $1^{\text {st }}$ column describes the indices the scene data points. The $d$ 's are the distances between the corresponding reference-scene points. Note that $d_{1}<d_{2}, d_{2}<d_{3}$, and so on.

For each point pi, the OICP considers the closest point from $\mathbf{M}$ as a correspondence pair. This might yield to correspondences that are surjective, where two or more scene points are assigned to a common reference point, as seen from the example of Table 1 where points $\mathrm{p} 2$ and $\mathrm{p} 3$ are assigned to the same point $\mathrm{m} 2$.

\begin{tabular}{|l|l:l:l:l|l|} 
& $\mathbf{m 1}$ & $\mathbf{m 2}$ & $\mathbf{m 3}$ & $\mathbf{m 4}$ & $\mathbf{m 5}$ \\
\hline $\mathbf{p 1}$ & $d 1$ & $d 5$ & $d 10$ & $d 22$ & $d 21$ \\
\hdashline $\mathbf{p} 2$ & $d 11$ & $d 3$ & $d 4$ & $d 18$ & $d 23$ \\
\hdashline $\mathbf{p 3}$ & $d 9$ & $d 2$ & $d 17$ & $d 20$ & $d 24$ \\
\hdashline $\mathbf{p 4}$ & $d 12$ & $d 13$ & $d 5$ & $d 6$ & $d 19$ \\
\hdashline $\mathbf{p 5}$ & $d 16$ & $d 25$ & $d 7$ & $d 14$ & $d 8$ \\
\hline
\end{tabular}

Table 1. Matching using OICP

Using the bijectivity property of the rotation, we propose to use a new evaluation for correspondence search, called comprehensive lookup matrix measure. This measure ensures that every selected point on the scene surface has a unique match in the reference surface.

The proposed algorithm is called Comprehensive ICP (CICP) algorithm. The CICP is different in that it sorts the distances in ascending order within the entire P-M distance matrix. Moreover, the point $\mathrm{m}_{j}$ is not considered to be a correspondence to $\mathrm{p} i$ if either $\mathrm{m} j$ or $\mathrm{pi}$ has been previously assigned a correspondence. This ensures that each point in the scene surface will have a different association in the reference surface. In this case, the points (p1-m1) corresponding to the minimum distance $(d 1)$ are chosen as a match. Without using the rows and columns of the previously selected pairs, the points (p3-m2) corresponding to the minimum distance $(d 2)$ of the resulting matrix are then chosen as another match, and so on (see Table 2).

\begin{tabular}{|l|l|l|l|l|l|}
\cline { 2 - 6 } \multicolumn{1}{c|}{} & $\mathbf{m 1}$ & $\mathbf{m} 2$ & $\mathbf{m} 3$ & $\mathbf{m 4}$ & $\mathbf{m 5}$ \\
\hline $\mathbf{p} 1$ & $d_{1}$ & $d_{5}$ & $d_{10}$ & $d_{22}$ & $d_{21}$ \\
\cline { 2 - 6 } $\mathbf{p} 2$ & $d_{11}$ & $d_{3}$ & $d_{4}$ & $d_{18}$ & $d_{23}$ \\
\cline { 2 - 6 } $\mathbf{p} 3$ & $d_{9}$ & $d_{2}$ & $d_{17}$ & $d_{20}$ & $d_{24}$ \\
\cline { 2 - 6 } $\mathbf{p} 4$ & $d_{12}$ & $d_{13}$ & $d_{5}$ & $d_{6}$ & $d_{19}$ \\
\cline { 2 - 6 } $\mathbf{p} 5$ & $d_{16}$ & $d_{25}$ & $d_{7}$ & $d_{14}$ & $d_{8}$ \\
\hline
\end{tabular}

Table 2. Matching using CICP

\section{OVERVIEW OF THE CICP ALGORITHM}

Assume that the given two surfaces to be registered can be described as point sets; the scene data points, $\mathbf{P}$, with $\mathrm{N}_{\mathrm{p}}$ points, $\left\{\mathrm{p}_{i}, i=1, \ldots, \mathrm{Np}\right\}$, and the reference data points, $\mathbf{M}$, with $\mathrm{N}_{\mathrm{m}}$ points, $\left\{\mathrm{m}_{i}, i=1, \ldots, \mathrm{N}_{\mathrm{m}}\right\}$. Depending on the accuracy of the constructed surfaces, $\mathrm{N}_{\mathrm{p}}$ is not necessarily equal to $\mathrm{N}_{\mathrm{m}}$. Furthermore, the point $\mathrm{p}_{i}$ of the scene surface does not necessarily represent $3 \mathrm{D}$ correspondence to the point $\mathrm{m}_{i}$ of the reference surface.

When the number of points in the two sets to be registered is not the same, the CICP algorithm considers the one with a smaller number of points as a scene data set to ensure bijectivity of the resulting correspondence pairs. To reduce computation time introduced by matrix search procedure, fast assignment algorithms can be used. The CICP algorithm can be summarized as follows:

I. Initialization:

1) Let the initial scene surface $\mathbf{P}_{0}$, be equal to $\mathbf{P}$.

2) Define the maximum number of iterations $\mathbf{k}_{\max }$.

3) Initialize the translation vector $\mathrm{Tt}_{0}$ and the rotation matrix $\operatorname{Tr}_{0}$ as follows:

II. Iterations:

1) For each point $p_{i} \in \mathbf{P},\left(i=1, \ldots, \mathrm{N}_{\mathrm{p}}\right)$, the algorithm computes the Euclidian distance to each point $m_{j} \in \mathbf{M},\left(j=1, \ldots, \mathrm{N}_{\mathrm{m}}\right)$. Then, for $\mathrm{N}_{\mathrm{p}}$ times, the algorithm.

A. looks for the location $(i, j)$ that corresponds to the min. distance in the current look up matrix,

B. assigns pi to $\mathrm{m} j$ as a correspondence pair,

C. removes this correspondence pair by eliminating the $i^{\text {th }}$ row and $j^{\text {th }}$ column

2) Using the selected correspondence pairs, compute the transformation, rotation $\left(\mathbf{T r}_{\mathrm{k}}\right)$ and translation $\left(\mathbf{T t}_{\mathrm{k}}\right)$, that minimises the mean square error (MSE) of these pairs

$$
\mathbf{M S E}=\frac{1}{\mathbf{N}_{\mathbf{p}}} \sum_{\mathbf{i}=1}^{\mathbf{N}_{\mathbf{p}}}\left\|y_{\mathbf{i}}-\mathrm{R}\left(p_{\mathbf{i}}\right)-\mathrm{T}\right\|^{2}
$$

The resulting transformation from the minimization of the above equation will be denoted as $\mathbf{T t}_{\mathrm{k}}$ and $\mathbf{T r}_{\mathrm{k}}$. This 
step provides also the minimum distances $d_{i, j}$ which correspond to the matched pairs.

$$
\mathbf{P}_{k}=\operatorname{Tr}_{k} \times \mathbf{P}_{0}+\mathrm{Tt}_{k} \text {. }
$$

and restart a new iteration if the change in the MSE is above a predefined threshold $\zeta$, and if the maximum number of iterations $\mathrm{k}_{\max }$ is not reached. If not, stop the iterations and exit.

\section{DATA DESCRIPTION}

In our experiments, we consider two sequences of the left ventricle of the heart acquired with two modalities on the same patient, at the same cardiac instants. The two examinations were carried out consecutively to ensure the best reproduction of cardiac deformations.

The previously introduced 4D echocardiography system consists of a rapid continuous rotation of a classical 2D scan head, which thus functions as a real 3D sensor. With sufficient number of rotations of the sensors (500 tours/min), the acquisitions are reduced to only one cardiac cycle. Because of the rotation of the sensor, the ultrasound beam sweeps a conic surface, where the contour of the LV is as visible as on standard planar images.

Starting from two hand traced contours, an automatic algorithm locates the LV contour on each image of the cardiac cycle. Then, a 4D interpolation method reconstructs the left ventricular surfaces within the cardiac cycle. The periodicities in space domain (closed convex contour of the LV) and in time domain (cardiac cycle) of the addressed problem were exploited by a fourdimensional harmonic modeling of the LV surface deformations [1]. Within a few seconds of acquisition and a few minutes of processing, the LV4D method provides closed surfaces of the LV similar to those presented in Fig.2.b.

The gated SPECT examination (at Orleans Hospital, France) was carried out on the same patient within two hours after the ultrasound examination. Under this protocol, the patient, at rest, receives an injection of 925 $\mathrm{MBq}$ of sestamibi marked with the $99 \mathrm{mTc}$. The examination was practiced one hour after the injection by means of a simple head camera DS7 provided by Sopha Medical Vision (SMV), equipped with a high resolution low energy collimator (HRLE). The acquisition, synchronized with the ECG and carried out with an energy window of width $20 \%$ regulated out of $140 \mathrm{KeV}$, comprises 32 projections of $45 \mathrm{~s}$ over $180^{\circ}$. Eight LV volumes data gated to the electro $\neg$ cardiogram were reconstructed within the cardiac cycle, in order to comply with the standard clinical investigations conditions obtained with the described camera.

The processing system was coupled with a PC computer used to transfer volumic information in the form of 64 images of $64 \times 64$ pixels coded with 16 bits. To obtain representative data of surfaces of the left ventricle, internal contours of the LV were extracted on the useful images from each of the eight considered volumes. Surfaces of the LV were then reconstructed by assembling all the samples obtained by volume, each one being validated by a cardiologist. Fig.2.a shows the 8 closed surfaces of the LV obtained with gated SPECT.

\section{RESULTS AND DISCUSSION}

\subsection{Registration of the LV sequences}

The registration of each of the two corresponding volumes was carried out by estimating the parameters of translation $(\mathrm{Tt})$ and rotation $(\mathrm{Tr})$. The rotation parameters, expressed in Euler angles $(\alpha, \beta, \gamma)$, are calculated starting from the rotation matrix (1) given by the algorithm CICP. Table 3 summarizes the rotation and translation ( $\tau x, \tau y, \tau z)$ parameters provided by the registration process.

\begin{tabular}{|c|c|c|c|c|c|c|}
\hline Surf. $\mathbf{N}^{\circ}$ & $\alpha$ & $\beta$ & $\gamma$ & $\tau_{\mathrm{x}}$ & $\tau_{\mathrm{y}}$ & $\tau_{\mathrm{z}}$ \\
\hline 1 & $104^{\circ}$ & $25.1^{\circ}$ & $13.9^{\circ}$ & -0.6 & -1.6 & -6.6 \\
\hline 2 & $103^{\circ}$ & $7.3^{\circ}$ & $4.7^{\circ}$ & -1.2 & -1.5 & -2.1 \\
\hline 3 & $98^{\circ}$ & $3.4^{\circ}$ & $-3.5^{\circ}$ & -0.3 & -3.2 & 0.2 \\
\hline 4 & $91^{\circ}$ & $39.0^{\circ}$ & $20.3^{\circ}$ & -1.8 & -3.8 & 1.2 \\
\hline 5 & $101^{\circ}$ & $1.1^{\circ}$ & $2.7^{\circ}$ & -2.2 & -3.5 & 0.8 \\
\hline 6 & $95^{\circ}$ & $0.0^{\circ}$ & $2.3^{\circ}$ & -2.8 & -2.3 & 0.6 \\
\hline 7 & $96^{\circ}$ & $2.2^{\circ}$ & $-1.4^{\circ}$ & 0.9 & -3.4 & -1.6 \\
\hline 8 & $89^{\circ}$ & $2.0^{\circ}$ & $-4.9^{\circ}$ & -1.2 & -5.4 & -0.3 \\
\hline
\end{tabular}

Table 3. Estimation of transformation parameters

Values of Table 3 were used to apply transformation on the 8 ultrasound LV surfaces of Fig.2b. The transformed ultrasound LV surfaces are represented in Fig.2c, registered with the corresponding 8 gated SPECT LV surfaces of Fig.2a. It can be observed that the surfaces of the $\mathrm{LV}$ are restored after a shift of about $90^{\circ}$ relatively to the axial view. This value is related to the acquisitions conditions which are dissimilar for the two modalities (left sidelying position during the ultrasonic examination and patient on the back for the gated SPECT imagery), and fully justify the registration step prior to comparison. Table 3 indicates that the rotation parameters around $\mathrm{X}$ and $\mathrm{Y}$ axes are evaluated correctly for LV shapes. Conversely, the determination of Euler angle $\gamma$ is more uncertain due to the rotational symmetry of the LV shape around the $\mathrm{Z}$ axis. Cardiologists consulted claimed the results obtained comply with regular medical requirements.

\subsection{Ultrasound LV4D data}

Fig.2d (last column) highlights the differences between the two sets of registered LV surfaces. Each closed surface is developed in a rectangular domain by transforming data form Cartesian to special coordinates, starting from the centre of gravity of each surface. Developed surfaces are then interpolated by a modified variation splines fitting (MVSF) algorithm [6] (based on a method previously presented by Szeliski [7]) for the reconstruction of the LV surfaces, and the signed difference is calculated between each pair of corresponding samples within the two surfaces. A color matrix, proportional to the range of the radial differences, is determined and projected on the reference gated SPECT surfaces. The color scale is unique for the 8 surfaces in order to allow the comparison between the surfaces. The direct color coding characterizes the 
differences between registered surfaces, by charting the distance between radial samples of the two surfaces.

From the medical point of view, the results presented in this study show that the surfaces provided by the LV4D method are close to the reference gated SPECT surfaces. Actually, for each of the 8 surfaces, the coding of colors remains in average around the homogeneous light blue green - yellow colors, indicating low radial distance between the two LV surfaces. Some higher errors appear for volumes $\mathrm{n}^{\circ} 4$ (dominant dark blue) and $\mathrm{n}^{\circ} 8$ (dominant red), probably due to lower signal to noise ratio, encountered occasionally during the acquisition as well as the reconstruction steps. The results obtained show a good registration between the two reconstructed sequences, and validate the ultrasound LV4D method as an alternative to the reference gated SPECT examination.

\section{RESULTS AND DISCUSSION}

The work presented in this paper describes a complete process that could be used to compare accurately two corresponding sphere-like shape closed surfaces. The method comprises three steps: 1) surface registration using an optimized version of the well-known ICP algorithm, 2) surface reconstruction using a reliable extension of the classical splines smoothing SVF method, 3) color representation of the radial distances between two corresponding surfaces, projected on the surface of reference. The approach proposed has been successfully applied to highlight the differences between 8 corresponding LV surfaces obtained from ultrasound and gated SPECT examinations. Finally, it was shown that the original LV4D method provides reconstructions of the LV surfaces equivalent to the surfaces obtained from gated SPECT, the "gold standard" examination technique in the field of dynamic cardiac reconstruction.

The study presented can be extended to measure the differences between closed or developed corresponding surfaces (images). It can then be used to compare different reconstructions of a unique surface, or to compare a unique reconstruction of different surfaces.

\section{ACKNOWLEDGMENTS}

The authors wish to thank the University of Orleans, France, the university of Sebha, Libya, and King Fahd University of Petroleum \& Minerals, KSA, for the support provided to carry this research.

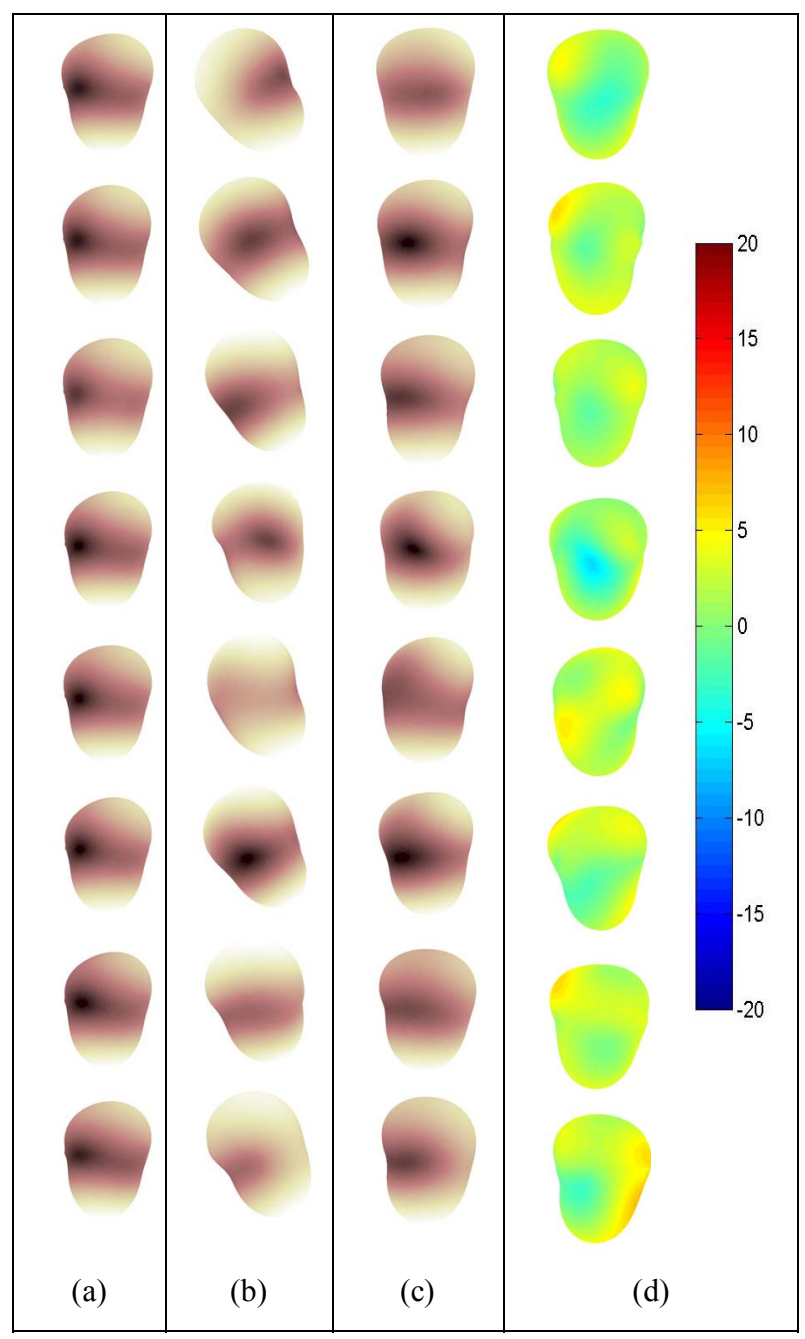

Fig. 2. Registration of two sequences of eight surfaces of the LV acquired from gated SPECT (a) and ultrasound (before registration (b) and after registration (c)).

Local comparison of the registered surfaces (d).

\section{REFERENCES}

[1] C. Bonciu, R. Weber and C. Léger, "4D reconstruction of the left ventricle during a single heart beat from ultrasound imaging", IEEE Transactions on Pattern Analysis and Machine Intelligence, vol. 19, no. 6, p. 401-412, 2001.

[2] J. Besl and H. D. McKay, "A method for registration of 3D shapes", Journal of IEEE Transactions on Pattern Analysis and Machine Intelligence, vol. 14, no. 2, 1992.

[3] B. Zitora, J. Flusser, "Image Registration Methods: a Survey", Image and Vision Computing, vol. 21, 2003.

[4] M. Andette, F. Ferrie, T. Peters 'An Algorithmic Overview of Surface Registration Techniques for Medical Imaging', Medical Image Analysis, vol. 4, pp. 201-217, 2000.

[5] S. Rusinkiewicz, M. Levoy, "Efficient variants of the ICP algorithm", 3rd International Conference on 3D Digital imaging and Modeling, Quebec, June 2001.

[6] A. Almhdie, C. Léger, M. Bergounioux and M. Deriche, "Improved VSF Algorithm for Smooth Surface Reconstruction from Sparse Medical Data", Journal of Computing \& Information Technology, vol. 15, no.2, 2007.

[7] R. Szeliski, "Fast surface interpolation using hierarchical basis functions", IEEE Transactions on Pattern Analysis and Machine Intelligence, vol. 12, no. 6, p. 513-528, 1990. 